\title{
Nonlinear dynamics of nanoelectromechanical cantilevers based on nanowire piezoresistive detection
}

\author{
N. Kacem ${ }^{1, \text { a }}$, S. Hentz ${ }^{2}$, S. Baguet ${ }^{3}$, and R. Dufour ${ }^{3}$ \\ 1 FEMTO-ST Institute - UMR CNRS 6174, 24, chemin de l'Épitaphe, F-25000 Besançon, France \\ 2 CEA/LETI - MINATEC, Grenoble, France \\ 3 Université de Lyon, CNRS, INSA-Lyon, LaMCoS UMR5259, F-69621, Villeurbanne, France
}

\begin{abstract}
The nonlinear dynamics of in-plane nanoelectromechanical cantilevers based on silicon nanowire piezoresistive detection is investigated using a comprehensive analytical model that remains valid up to large displacements in the case of electrostatic actuation. This multiphysics model takes into account geometric, inertial and electrostatic nonlinearities as well as the fringing field effects which are significant for thin resonators. The bistability as well as multistability limits are considered in order to provide close-form expressions of the critical amplitudes. Third order nonlinearity cancellation is analytically inspected and set via an optimal DC drive voltage which permits the actuation of the NEMS beyond its critical amplitude. It may result on a large enhancement of the sensor performances by driving optimally the nanocantilever at very large amplitude, while suppressing the hysteresis.
\end{abstract}

\section{Introduction}

Nanoelectromechanical systems (NEMS) are drawing interest from the scientific community for a wide range of applications due to their unique properties. Nanocantilevers are among those of the possible NEMS realizations that offer access to fundamental resonant frequencies in the microwaves and active masses in the femtograms. Nanocantilever have been proposed for ultrafast sensors and actuators, signal processing components, quantum computing [1] as well as ultra sensitive force [2] and mass [3] detection.

Actually, it is a challenge to optimize NEMS mass sensors in order to achieve high resolutions. Although the linear design optimization and mechanical transduction gain of the devices have been thoroughly studied, the drive power has always been a priori limited by the onset of nonlinearities. Indeed, driving the cantilever at large oscillation amplitude leads to better signal to noise ratio (SNR) and, thus, simplifies the design of the electronic feedback loop. However, doing so in the nonlinear regime reduces the sensor performances since the frequency instability of a nonlinear resonator is proportional to its oscillation amplitude. Moreover, even when NEMS resonators are used as oscillators in closed-loop, a large part of noise mixing [4] due to nonlinearities drastically reduces their dynamic range and alters their detection limit.

The nonlinear dynamics of cantilevers have received considerable attention because of their importance in many engineering applications. Crespo da Silva and Glynn $[5,6]$ derived a set of integro-partial-differential equations governing flexural-flexural-torsional motions of inextensional beams, including geometric and inertia nonlinearities. They used these equations and the method of multiple scales to ascertain the importance of the geometric terms for the

\footnotetext{
a e-mail: najib.kacem@femto-st.fr
}

lower modes, especially the first mode. These equations have been also used for several investigations such as the non-planar responses of cantilevers to principal parametric and primary resonant excitations [7,8], the nonlinear response of an inextensional beam to a primary resonant excitation of one of its flexural modes when the first torsional frequency is of the same order as the lower flexural frequencies $[9,10]$, as well as the nonlinear non-planar response of cantilever inextensional metallic beams to a parametric excitation of order two its flexural modes [11].

This paper proposes a method to overcome the physical limitations in NEMS mass sensors when operating beyond their critical amplitudes. Based on the nonlinear dynamics of nanomechanical cantilevers, the main idea is to provide simple analytical tools for NEMS designers in order to optimize mass resonant sensors designs and enhance their performances for precision measurement applications such as mass spectrometry.

In [12], a reduced-order analytical model was developed to investigate the nonlinear dynamics of NEMS resonant cantilever electrostatically actuated and based on capacitive detection. In this paper, NEMS cantilever based on silicon nanowire piezoresistive detection are considered including both mechanical and electrostatic nonlinearities. Due to the geometry of the device, a specific Galerkin decomposition procedure based on piecewise basis functions is used and the resonance under primary excitation is investigated by means of a perturbation technique. The resulting analytical model enables the capture of the main nonlinear phenomena including the mixed hardening-softening behavior $[13,14]$. Thus, close-form solutions of the critical amplitude in the hardening as well as the softening case are deduced and the optimal DC driving voltage expression in function of the design parameters is provided. Hence, the model can be used by NEMS designers as a practical tool for the enhancement of resonant sensor performances based on the nonlinearity cancellation. 


\section{Device description}

The NEMS is composed of a fixed-free lever beam linked to two piezoresistive gauges at a distance $d=0.15 \mathrm{l}$ from its fixed end. This value was chosen to maximize the stress inside the gauges due to the cantilever motion (Figure 1). The NEMS cantilever is actuated electrostatically at the primary resonance of its first linear undamped mode shape. The cantilever oscillation induces stress inside the piezoresistive gauges and the collected strain is transduced into a resistance variation due to the piezoresistive effect. Thus, the sensor frequency response is obtained via a piezoresistive read-out perfectly decoupled from the capacitive actuation of the resonator. Such a device can be used either as a mass or a gas sensor. Indeed, the amount of molecules absorbed by the surface of the cantilever changes its effective mass which lowers its resonance frequency. By evaluating the frequency shift, the mass of the added species can be estimated. Driven below its critical amplitude, This device

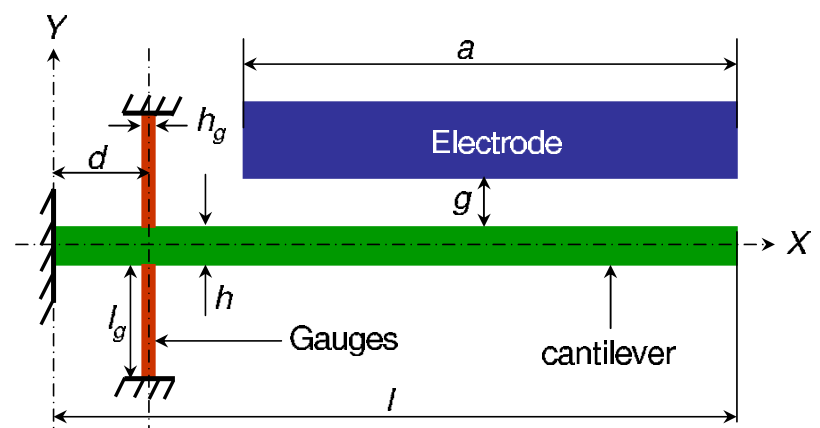

Fig. 1. Resonant nanocantilever based on piezoresistive detection.

has shown promising performances in terms of frequency stability, dynamic range, and achievable mass resolution of few zeptograms [15]. Unlike the stand-alone NEMS described in [16], it uses CMOS based fabrication and is therefore fully compatible with very large scale integration (VLSI) of NEMS. Thus, such device is transferable to semiconductor foundries on $200 \mathrm{~mm}$ wafer for the future. Nevertheless, further dynamic range optimization for lower mass resolution could potentially be based on the nonlinear dynamics of such mechanical structure.

\section{Model}

\subsection{Equation of motion}

A variational approach, based on the extended Hamilton principle $[5,6,17,18]$ is fallowed in order to derive the nonlinear equations of motion describing the flexural vibration of the device sketched in Figure 1. The cantilever bending deflection $\tilde{w}_{j}$ is decomposed into $\tilde{w}_{0}$ for $\tilde{s} \in[0, d]$ and $\tilde{w}_{1}$ for $\tilde{s} \in[d, l]$.

$$
\begin{aligned}
& E I_{c}\left\{\tilde{w}_{j}^{\prime \prime \prime \prime}+\left[\tilde{w}_{j}^{\prime}\left(\tilde{w}_{j}^{\prime} \tilde{w}_{j}^{\prime \prime}\right)^{\prime}\right]^{\prime}\right\}+\rho b h \ddot{\tilde{w}}_{j}+\tilde{c} \dot{\tilde{w}}_{j}= \\
& -\frac{1}{2} \rho b h\left\{\tilde{w}_{j}^{\prime} \int_{(1-j) d+j l}^{s}\left[\frac{\partial^{2}}{\partial \tilde{t}^{2}} \int_{j d}^{s_{1}}\left(\tilde{w}_{j}^{\prime}\right)^{2} d s_{2}\right] d s_{1}\right\}^{\prime}
\end{aligned}
$$

$$
+\frac{j}{2} \varepsilon \frac{C_{n} b[V d c+V a c \cos (\tilde{\Omega} \tilde{t})]^{2}}{\left(g-\tilde{w}_{j}\right)^{2}} H(s+a-l)
$$

where $j \in\{0,1\}, s$ is the arclength, $E$ and $I_{c}$ are the Young's modulus and moment of inertia of the nanocantilever cross section. $l$ and $h$ are its length and its width. $b$ is the device thickness, $\rho$ is the material density, $g$ is the capacitor gap width, and $\varepsilon$ is the dielectric constant of the gap medium. The last term in Equation (1) represents an approximation of the electrostatic force assuming a partial distribution along the nanobeam length. $H$ is a Heaviside function and $C_{n}$ is the fringing field coefficient [19]. The boundary conditions are:

$$
\begin{array}{r}
\tilde{w}_{0}(0, \tilde{t})=\tilde{w}_{0}{ }^{\prime}(0, \tilde{t})=\tilde{w}_{1}{ }^{\prime \prime}(l, \tilde{t})=\tilde{w}_{1}{ }^{\prime \prime \prime}(l, \tilde{t})=0 \\
\tilde{w}_{0}(d, \tilde{t})-\tilde{w}_{1}(d, \tilde{t})=\tilde{w}_{0}{ }^{\prime}(d, \tilde{t})-\tilde{w}_{1}^{\prime}(d, \tilde{t})=0 \\
\tilde{w}_{0}{ }^{\prime \prime}(d, \tilde{t})-\tilde{w}_{1}{ }^{\prime \prime}(d, \tilde{t})=-\frac{I_{g}}{I_{c} l_{g}} \tilde{w}_{0}{ }^{\prime}(d, \tilde{t}) \\
\tilde{w}_{0}{ }^{\prime \prime \prime}(d, \tilde{t})-\tilde{w}_{1}^{\prime \prime \prime}(d, \tilde{t})=-\frac{2 h_{g} b}{I_{c} l_{g}} \tilde{w}_{0}(d, \tilde{t})
\end{array}
$$

where $\tilde{t}$ is time, $h_{g}$ and $I_{g}$ are the width and the moment of inertia of the gauge cross section. Equations (4) and (5) are obtained by writing the force and torque moment equilibrium equations at the point $s=d$.

$$
\begin{array}{r}
\left.T_{s c}\right|_{d}+\left.2 T_{a g}\right|_{d}=-\left.\frac{\partial M_{b c}}{\partial s}\right|_{d}+\left.2 E b h_{g} \epsilon_{g}\right|_{d}= \\
E I_{c}\left[\tilde{w}_{0}^{\prime \prime \prime}(d, \tilde{t})-\tilde{w}_{1}^{\prime \prime \prime}(d, \tilde{t})\right] \\
+2 E b h_{g} \frac{\tilde{w}_{0}(d, \tilde{t})}{l_{g}}=0 \\
\left.M_{b c}\right|_{d}+\left.M_{g}\right|_{d}=E I_{c}\left[\tilde{w}_{0}{ }^{\prime \prime}(d, \tilde{t})-\tilde{w}_{1}{ }^{\prime \prime}(d, \tilde{t})\right] \\
-E I_{g} \frac{\tilde{w}_{0}{ }^{\prime}(d, \tilde{t})}{l_{g}}=0
\end{array}
$$

where $T_{s c}$ is the shear force applied to the cantilever, $M_{b c}$ is its bending moment, $T_{a g}$ is the axial force applied to the gauges and $M_{g}$ is its corresponding torque moment.

\subsection{Normalization}

Let the nondimensional variables be:

$$
w_{j}=\frac{\tilde{w}_{j}}{g}, \quad x=\frac{s}{l}, \quad t=\frac{\tilde{t}}{\tau}
$$

where $\tau=\frac{2 l^{2}}{h} \sqrt{\frac{3 \rho}{E}}$. Substituting Equation (8) into Equations (1-5), gives:

$$
\begin{array}{r}
w_{j}^{i v}+\ddot{w}_{j}+c \dot{w}_{j}+\delta_{1}\left[w_{j}^{\prime}\left(w_{j}^{\prime} w_{j}^{\prime \prime}\right)^{\prime}\right]^{\prime}= \\
-\delta_{2}\left\{w_{j}^{\prime} \int_{(1-j) \frac{d}{l}+j}^{x}\left[\frac{\partial^{2}}{\partial t^{2}} \int_{j \frac{d}{l}}^{x_{1}}\left(w_{j}^{\prime}\right)^{2} d x_{2}\right] d x_{1}\right\}^{\prime} \\
+j \delta_{3} \frac{V_{d c}}{V_{a c}} \frac{\left[1+\frac{V_{a c}}{V_{d c}} \cos (\Omega t)\right]^{2}}{\left(1-w_{j}\right)^{2}} H\left(x+\frac{a}{l}-1\right) \\
w_{0}(0, t)=w_{0}^{\prime}(0, t)=w_{1}^{\prime \prime}(1, t)=w_{1}^{\prime \prime \prime}(1, t)=0
\end{array}
$$




$$
\begin{array}{r}
w_{0}\left(\frac{d}{l}, t\right)-w_{1}\left(\frac{d}{l}, t\right)=w_{0}^{\prime}\left(\frac{d}{l}, t\right)-w_{1}^{\prime}\left(\frac{d}{l}, t\right)=0 \\
w_{0}^{\prime \prime}\left(\frac{d}{l}, t\right)-w_{1}^{\prime \prime}\left(\frac{d}{l}, t\right)=-\frac{I_{g} l}{I_{c} l_{g}} w_{0}^{\prime}\left(\frac{d}{l}, t\right) \\
w_{0}^{\prime \prime \prime}\left(\frac{d}{l}, t\right)-w_{1}^{\prime \prime \prime}\left(\frac{d}{l}, t\right)=-\frac{2 h_{g} b l^{3}}{I_{c} l_{g}} w_{0}\left(\frac{d}{l}, t\right)
\end{array}
$$

The parameters appearing in Equation (9) are:

$$
\begin{gathered}
c=\frac{\tilde{c} l^{4}}{E I_{c} \tau}, \quad \delta_{1}=\left[\frac{g}{l}\right]^{2}, \quad \delta_{2}=\frac{1}{2}\left[\frac{g}{l}\right]^{2} \\
\delta_{3}=6 V_{a c} V_{d c} \frac{\varepsilon l^{4}}{E h^{3} g^{3}}, \quad \Omega=\tilde{\Omega} \tau
\end{gathered}
$$

\subsection{Reduced-order model}

Assuming high quality factors $\left(10^{3}-10^{4}\right)$, the static deflection can be neglected with respect to the dynamic deflection. A reduced-order model is generated by modal decomposition transforming the continuous problem (9) into a multi-degree-of-freedom system consisting in ordinary differential equations in time. The undamped linear mode shapes of the NEMS are used as basis functions in the Galerkin procedure, and the deflection is approximated by:

$$
w(x, t)=\sum_{k=1}^{n} a_{k}(t) \phi_{k}(x)
$$

where $n$ is the number of the first modes retained in the reduced-order model and $a_{k}(t)$ is the $k^{\text {th }}$ generalized coordinate. Since the boundary conditions depend on the position along the cantilever, the linear undamped bending modes $\phi_{k}$ of the device are defined as piecewise functions:

$$
\begin{aligned}
& x \in\left[0, \frac{d}{l}\right] \quad \phi_{0 k}(x)=A_{0 k} \cos \sqrt{\lambda_{k}} x+B_{0 k} \sin \sqrt{\lambda_{k}} x \\
& +C_{0 k} \cosh \sqrt{\lambda_{k}} x+D_{0 k} \sinh \sqrt{\lambda_{k}} x(16) \\
& x \in\left[\frac{d}{l}, 1\right] \quad \phi_{1 k}(x)=A_{1 k} \cos \sqrt{\lambda_{k}} x+B_{1 k} \sin \sqrt{\lambda_{k}} x \\
& +C_{1 k} \cosh \sqrt{\lambda_{k}} x+D_{1 k} \sinh \sqrt{\lambda_{k}} x
\end{aligned}
$$

Here, $\lambda_{k}$ is the $k^{\text {th }}$ natural frequency of the mechanical structure. $\phi_{0 k}$ and $\phi_{1 k}$ satisfy the boundary conditions defined in Equations (10-13). The first mode is then:

$$
\begin{array}{rl}
\phi_{01}(x)= & 0.00038 \cos (2.21 x)-0.0035 \sin (2.21 x) \\
-0 & .00038 \cosh (2.21 x)+0.0035 \sinh (2.21 x) \\
\phi_{11}(x) & =-1.284 \cos (2.21 x)+0.401 \sin (2.21 x) \\
& +1.413 \cosh (2.21 x)-1.206 \sinh (2.21 x)
\end{array}
$$

The electrostatic force in Equation (9) is expanded in a fifth order Taylor series to capture the mixed behavior $[13,14$, 12] physically significant in nonlinear nanoelectromechanical resonators. Then, Equation (15) is substituted into the resulting equation and the outcome is multiplied by $\phi_{k}$ and integrated from $x=0$ to 1 for $k \in[1, n] \cap \mathbb{N}$. Thus, a system of coupled ordinary differential equations in time is obtained.
Assuming that the first mode is the dominant mode of the system, the investigation can be restricted to the case $n=1$. Then, Equation (9) becomes:

$$
\begin{array}{r}
a_{1}^{\prime \prime}+c a_{1}^{\prime}+a_{1}\left(23.68+14.97 \delta_{2} a_{1}^{\prime 2}\right)+126.12 \delta_{1} a_{1}^{3} \\
-\delta_{3}\left[1.43 \cos (\Omega t)+\left(0.36 \frac{V_{a c}}{V_{d c}}+0.72 \frac{V_{d c}}{V_{a c}}\right)\right] \\
+14.97 \delta_{2} a_{1}^{2} a_{1}^{\prime \prime}-\delta_{3}\left[\frac{V_{a c}}{V_{d c}}+2 \frac{V_{d c}}{V_{a c}}+4 \cos (\Omega t)\right] a_{1} \\
-\delta_{3}\left[2.4 \frac{V_{a c}}{V_{d c}}+4.81 \frac{V_{d c}}{V_{a c}}+9.62 \cos (\Omega t)\right] a_{1}^{2} \\
-\delta_{3}\left[5.53 \frac{V_{a c}}{V_{d c}}+11.05 \frac{V_{d c}}{V_{a c}}+22.1 \cos (\Omega t)\right] a_{1}^{3} \\
-\delta_{3}\left[12.44 \frac{V_{a c}}{V_{d c}}+24.89 \frac{V_{d c}}{V_{a c}}+49.77 \cos (\Omega t)\right] a_{1}^{4} \\
-\delta_{3}\left[27.7 \frac{V_{a c}}{V_{d c}}+55.4 \frac{V_{d c}}{V_{a c}}+110.82 \cos (\Omega t)\right] a_{1}^{5} \\
-\delta_{3} \frac{V_{a c}}{V_{d c}}\left[5.53 a_{1}^{3}+12.44 a_{1}^{4}+27.7 a_{1}^{5}\right] \cos (2 \Omega t) \\
-\delta_{3} \frac{V_{a c}}{V_{d c}}\left[0.36+a_{1}+2.4 a_{1}^{2}\right] \cos (2 \Omega t)=0
\end{array}
$$

Assuming that the amplitude $A(t)$ and the phase $\beta(t)$ of the solution $a_{1}(t)$ are slowly time-varying functions, the averaging method is used in order to solve the nonlinear Mathieu-Duffing Equation (20). It permits the transformation of the reduced-order nonlinear second order Equation (20) into two first order nonlinear ordinary differential equations that describe the amplitude and phase modulation of the system frequency response.

For $V_{a c}<<V_{d c}$, the second harmonic terms are negligible. The resulting phase and amplitude averaged equations over the period $\frac{2 \pi}{\Omega}$ and around the primary resonance $\left(\Omega=\lambda_{1}+\xi \sigma\right)$ are:

$$
\begin{array}{r}
\dot{A}=-\xi \frac{\delta_{3} \sin \beta}{\lambda_{1}}\left(0.716+1.2 A^{2}+3.11 A^{4}\right) \\
-\xi \frac{c}{2} A+O\left(\xi^{2}\right) \\
\dot{\beta}=\xi\left[\frac{11.84}{\lambda_{1}}+\frac{47.3 \delta_{1} A^{2}}{\lambda_{1}}-\frac{4.14 \delta_{3} V_{d c} A^{2}}{V_{a c} \lambda_{1}}-\frac{\lambda_{1}}{2}\right] \\
+\xi\left[\frac{\delta_{3} \cos \beta}{\lambda_{1}}\left(\frac{0.716}{A}-15.55 A^{3}\right)-\frac{V_{d c} \delta_{3}}{V_{a c} \lambda_{1}}\right] \\
-\xi\left[\frac{17.32 \delta_{3} V_{d c} A^{4}}{V_{a c} \lambda_{1}}+3.74 \delta_{2} \lambda_{1} A^{2}\right] \\
-\frac{3.61 \xi A \delta_{3} \cos \beta}{\lambda_{1}}+O\left(\xi^{2}\right)
\end{array}
$$

The steady-state motions occur when $\dot{A}=\dot{\beta}=0$, which corresponds to the singular points of Equations (21) and (22).

\section{Nonlinear behaviors}

The normalized displacement $W_{\max }$ with respect to the gap at the middle of the beam and the drive frequency $\Omega$ can be expressed as a function of the phase $\beta$. Thus, the frequency 
response curve can be plotted parametrically. Several analytical simulations were performed using the following parameters: $l=5 \mu \mathrm{m}, b=160 \mathrm{~nm}, h=300 \mathrm{~nm}, l_{g}=500 \mathrm{~nm}$, $h_{g}=80 \mathrm{~nm}$ and $a=350 \mu \mathrm{m}$. The gap $\mathrm{g}$, the quality factor $Q$, the $D C$ and $A C$ voltages were used for parametric analysis.

\subsection{Hardening behavior}

The large deformations of the cantilever give rise to geometric nonlinearities due to nonlinear curvature and/or mid-plane stretching, leading to nonlinear strain-displacement relations. In other words, for cantilever beams, the principal mechanical nonlinearity results from a geometric effect: as the cantilever deflects its local stiffness and effective mass increase.

When this nonlinearity dominates the resonator dynamics, the frequency response peak is hysteretic and shifted to the high frequencies as shown in Figure 2. This so called "spring hardening" is the most classical effect observed in clamped-clamped resonators electrostatically actuated [20, 21].

As shown in figure 2, the critical amplitude is the oscillation amplitude $A_{c}$ above which bistability occurs. Thus, $A_{c}$ is the transition amplitude from the linear to the nonlinear behavior.

In order to build a close-form solution of the critical mechanical amplitude, Equations (21) and (22) are rewritten when the electrostatic nonlinearities (nonlinear terms proportional to $\delta_{3}$ ) are negligible. Then, the frequency response is expressed in its parametric form with respect to the phase $\beta$ as follows:

$$
\begin{aligned}
& \Omega=f_{1}(\beta) \\
& A=f_{2}(\beta)
\end{aligned}
$$

Mathematically, $A_{c m}$ is defined as the oscillation amplitude for which the equation $\frac{d \Omega}{d \beta}=0$ (infinite slope) has a unique solution $\beta_{c m}=\frac{\pi}{3}$. Thus, the critical electrostatic force is deduced and then substituted into Equation (24) at the point $\beta=\frac{\pi}{2}$ and multiplied by the coefficient of the first linear undamped mode shape $\phi_{1}$ at the free point of the beam. Finally, we obtain the following close-form solution of the critical mechanical amplitude:

$$
A_{c m}=6.3 \frac{l-d}{\sqrt{Q}}
$$

This expression is the same as on [12], but the cantilever length $l$ replaced by $l-d$. This confirms that the nonlinearities acting between the fixed end of the cantilever and the nanogauges are negligible since the sensor vibrations in this part are close to zero for the first linear undamped mode shape. Therefore, the NEMS dynamics is equivalent to a resonant nanocantilever of length $l-d$.

\subsection{Softening behavior}

In order to increase the softening electrostatic nonlinearities, the resonator designs must display narrow gaps with

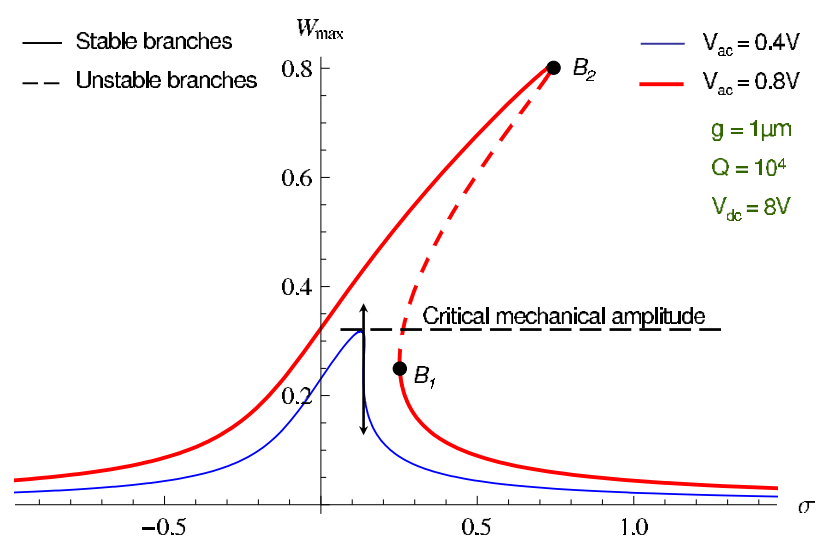

Fig. 2. Predicted hardening and critical mechanical behaviors of the typical resonant piezoresistive NEMS mass sensor described in Figure 1. $\sigma$ is the detuning parameter, $W_{\max }$ is the displacement of the beam normalized by the gap $g$ at its free end and $\left\{B_{1}, B_{2}\right\}$ are the bifurcation points.

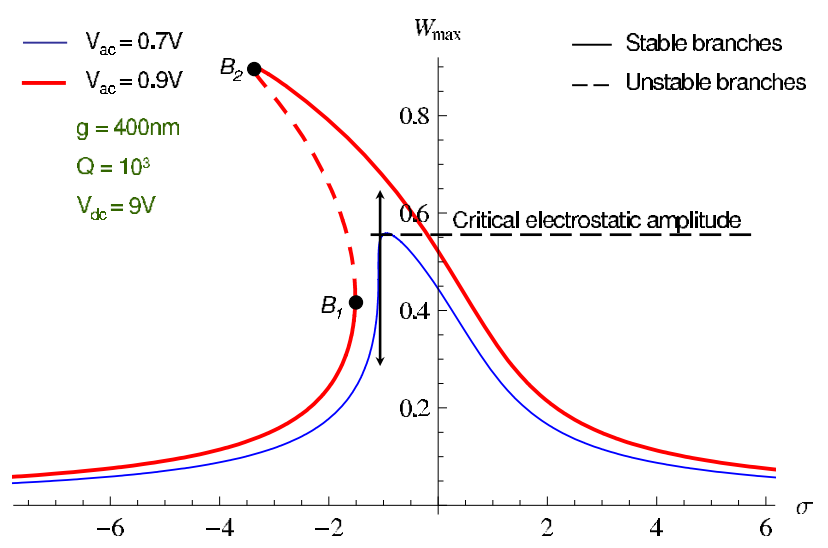

Fig. 3. Predicted softening and critical electrostatic behaviors of the typical resonant piezoresistive NEMS mass sensor described in Figure $1 . \sigma$ is the detuning parameter, $W_{\max }$ is the displacement of the beam normalized by the gap $g$ at its free end and $\left\{B_{1}, B_{2}\right\}$ are the bifurcation points.

respect to the cantilever width. Combined with a relatively low quality factor, this leads to a negative global Duffing nonlinearity and thus a softening behavior as shown in Figure 3. In this nonlinear regime, the frequency response curve is hysteretic and shifted to the low frequencies. Unlike clamped-clamped beam resonators, the softening behavior is easily reachable in NEMS resonant cantilevers.

In this case, the critical electrostatic amplitude can be deduced in the same way as the mechanical one. Here, the mechanical nonlinearities are negligible (terms proportional to $\delta_{1}$ and $\delta_{2}$ ). By considering only nonlinear terms up to the third order, while neglecting the parametric terms and the terms proportional to $V_{a c}^{2}$, the critical electrostatic amplitude is deduced as follows:

$$
A_{c e}=\frac{2 * 10^{9} h g^{\frac{5}{2}}}{(l-d) \sqrt{Q} V_{d c}}\left(\frac{7.5 * 10^{7} h^{2}}{(l-d)^{4}}-\frac{3.8 V_{d c}^{2}}{10^{15} g^{3} h}\right)^{\frac{1}{4}}
$$

Again, Equation (26) is in good agreement with the close form solution of the critical electrostatic amplitude developed in [12] in the case of NEMS cantilevers, which confirms that the electrostatic nonlinearities are acting only 


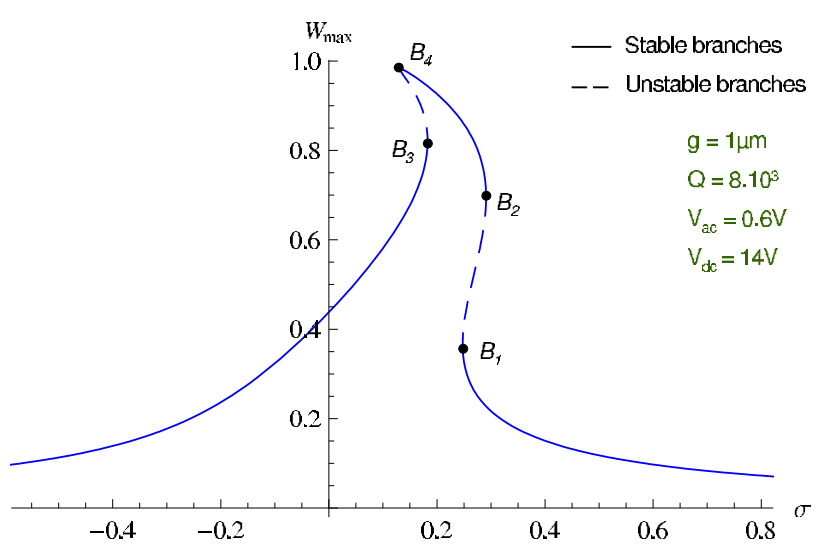

Fig. 4. Predicted mixed behavior of the typical resonant piezoresistive NEMS mass sensor described in Figure 1. $\sigma$ is the detuning parameter, $W_{\max }$ is the displacement of the beam normalized by the gap $g$ at its free end and $\left\{B_{1}, B_{2}, B_{3}, B_{4}\right\}$ are the bifurcation points.

on the sensor part comprised between the gauges and the free end of the device.

\subsection{Mixed behavior}

Both mechanical and electrostatic nonlinearities are always operating into the system. However, in some configurations one kind of nonlinearity is negligible with respect to the second one. Practically, when $h / g<<1$ and for a high quality factor, the dynamics is dominated by the hardening nonlinearities and in the opposite case, the frequency response is nonlinearly softening. Between these two configurations and for the typical resonant piezoresistive mass sensor shown in Figure 1, a mixed hardening-softening behavior is inescapable (Figure 4). The mixed behavior is characterized by a four-bifurcation points frequency response. Moreover, Kacem et al [14] demonstrated in the case of clamped-clamped resonators, an electrostatic mechanism which enables the bifurcation topology tuning of the mixed behavior so that the bifurcation point $B_{1}$ can be moved to a lower frequency than $B_{3}$. This leads to a multistability with 5 possible amplitudes for a given frequency. It is highly unstable and thus undesirable for MEMS and NEMS designers.

\section{Engineering optimization}

Figure 5 shows several predicted forced frequency response of the resonant piezoresistive mass sensor. Clearly, the analytical model enables the capture of the main nonlinear regimes in the resonator dynamics and describes the competition between the mechanical hardening and the electrostatic softening behaviors. As shown in Figure 5, when $g<<h$, the mechanical nonlinearities are negligible with respect to the electrostatic nonlinearities. Then, the NEMS forced frequency curve displays a softening behavior (red curve of Figure 5) and the critical amplitude is given by Equation (26) which depends on the quality factor $Q$, the cantilever width $h$, the gap $g$, the DC voltage $V_{d c}$ and the distance between the piezoresistive nanogauges and the cantilever free end $l-d$. In this case, the open-loop stability

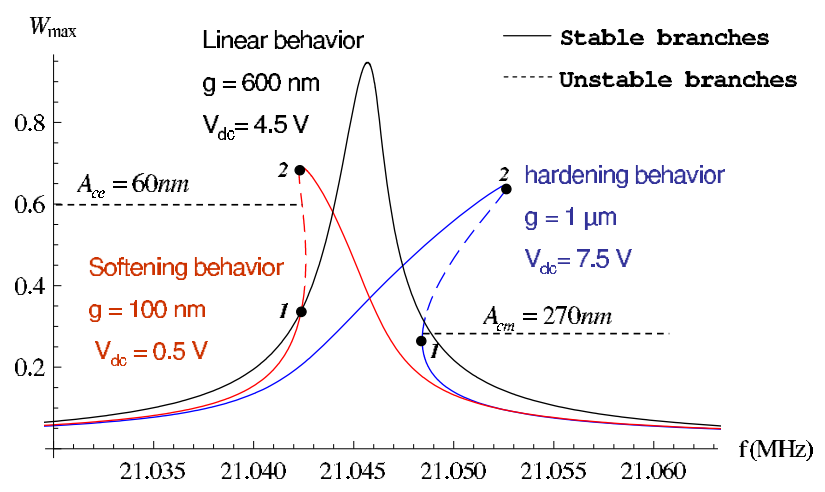

Fig. 5. Predicted forced frequency responses of the resonant piezoresistive device presented in Figures 1 for a quality factor $Q=10^{4}$ and $V_{a c}=0.1 V_{d c}$. $W_{\max }$ is the displacement of the beam normalized by the gap $g$ at its free end.

of the NEMS resonant sensor is limited by an oscillation amplitude around $60 \mathrm{~nm}$.

If $g>>h$, the electrostatic nonlinearity is negligible with respect to the mechanical one. Then, The NEMS forced frequency curve displays a hardening behavior (see Figure 5) and the critical amplitude is given by Equation (25) which only depends on the quality factor $Q$ and the distance between the piezoresistive nanogauges and the cantilever free end $l-d$. In this case, the open-loop stability of the NEMS resonant sensor is limited by an oscillation amplitude around $270 \mathrm{~nm}$ : more than four times higher than the previous case. Thus the resolution is enhanced by a factor $\Pi_{e n h}=4$ compared to the first case.

Hence, designing NEMS cantilevers displaying softening behaviors is disadvantageous and can significantly alter the sensor resolution especially when this supposes that we are able to fabricate structures with very small gaps which is rather difficult. Indeed, assuming that the upper bound limit which is the pull-in occurs at an amplitude of the gap order, even if the cantilever can vibrate linearly up to very high amplitudes comparable to the gap, the sensor performances can be altered due to its small dimensional amplitude limited by the gap. In other words, enhancing the dimensionless critical amplitude (softening behavior in Figure 5) is not important when the gap is significantly reduced.

The optimal gap is $g_{p}=600 \mathrm{~nm}$ for which the mechanical and the electrostatic nonlinearities are balanced which permits the linearization of the frequency response as shown in Figure 5 (linear behavior in Figure 5). For this design which is technologically feasible, the resolution is enhanced by a factor $\Pi_{e n h}=9$, compared to first case.

In practice and since cantilever designs are generally set by technological choices and sensor specifications, it is more pertinent and useful to provide an optimal $D C$ drive voltage rather than an optimal gap. It leads to an electrostatic mechanism which enables the hysteresis suppression by nonlinearity cancellation.

Moreover, It has been identified that the mixed behavior [14] in cantilevers is less pronounced than in electrostatically driven clamped-clamped beams [12]. Consequently, the validity domain of the third order nonlinearity cancellation in cantilevers is potentially larger. Thus, the hysteresis suppression condition can be written as

$$
A_{c_{m}}=A_{c_{e}}
$$


Hence, while neglecting the ohmic losses [22] and therefore assuming a constant quality factor $Q$, the optimal $D C$ drive voltage is

$$
V_{d c_{O P}}=\sqrt{\begin{array}{c}
\frac{1}{2} \sqrt{\frac{1.65 * 10^{39} g^{14} h^{6}}{(l-d)^{16}}+\frac{3.2 * 10^{42} g^{10} h^{6}}{(l-d)^{12}}} \\
-\frac{8.1 * 10^{19} g^{7} h^{3}}{(l-d)^{8}}
\end{array}}
$$

In the particular case of Figure 5, the mechanical critical amplitude is $A_{c_{m}}=45 \% \mathrm{~g}$. When $g=600 \mu \mathrm{m}$ and for a quality factor of $Q=10^{4}$, the optimal $D C$ drive voltage is around $4.5 \mathrm{~V}$. At this voltage, as shown by the black curve of Figure 5, the peak amplitude is linear and beyond the critical amplitude $\left(A_{\text {peak }}>90 \% \mathrm{~g}\right)$. Therefore, the enhancement rate of the sensor performance is $\left(\frac{A_{\text {peak }}}{A_{c}}\right)>2$.

Remarkably, the electrostatic critical amplitude is independent of the $A C$ voltage. This is due to the use of a low $A C$ voltage compared to the $D C$ voltage for the cantilever actuation which makes the contribution of $V_{a c}$ in the electrostatic Duffing term negligible. Hence, in this configuration, the compensation of the nonlinearities is independent on the $A C$ voltage. This interesting result makes possible the enhancement of the piezoresistive NEMS sensor performances up to very high displacements comparable to the gap in the case of an electrostatic actuation by increasing the $A C$ voltage, and limited by an upper bound instability such as the pull-in [23].

\section{Conclusion}

In this paper, the nonlinear dynamics of a resonant piezoresistive NEMS mass sensor under its primary resonance has been investigated using a multiphysics analytical model which includes both mechanical and electrostatic nonlinearities. The model is based on the modal decomposition using a specific Galerkin procedure with piecewise basis functions combined with a perturbation technique, namely the averaging method. The model enables the capture of the main nonlinear phenomena including the mixed behavior. The bistability as well as multistability limits of the NEMS dynamics have been analytically investigated and close-form solutions of the critical mechanical and electrostatic amplitudes have been provided. These expressions have been validated with respect to the model developed in [12]. Finally, the analytical expression of the optimal $D C$ drive voltage has been extracted. Besides, it permits the control of the nonlinearities via an electrostatic mechanism in order to drive the NEMS resonator linearly beyond its critical amplitude. This rule could be a new device specification for NEMS designers by defining the appropriate polarization corresponding to the operating domain of the third order nonlinearity cancellation. As a consequence, the sensor sensitivity could significantly be enhanced. Indeed, the carrier power of the resonator is largely increased at large amplitudes and keeping a linear behavior may prevent most of noise mixing [4]. In practice, this technique of hysteresis suppression could potentially enhance the NEMS performances and provide mass resolutions sufficient to detect individual nanoparticules and biomolecules in real time and VLSI fashion for mass spectrometry applications [24,25].

\section{References}

1. S. Bose and G.S. Agarwal, New Journal of Physics, 8(3):34, (2006).

2. Y.G. Jiang, T. Ono, and M. Esashi, Measurement Science and Technology, 19(8):084011, 2008.

3. M. Li, H.X. Tang, and M.L. Roukes, Nature Nanotechnology, 2:114-120, (2007).

4. V. Kaajakari, J. K Koskinen, and T. Mattila, IEEE Transactions on Ultrasonics, Ferroelectrics and Frequency Control, 52(12):2322-2331, (2005).

5. M.R.M. Crespo Da Silva and C.C. Glynn, Journal of Structural Mechanics, 6:437-448, (1978).

6. M.R.M. Crespo Da Silva and C.C. Glynn, Journal of Structural Mechanics, 6:449-461, (1978).

7. A.H. Nayfeh and P.F. Pai, International Journal of NonLinear Mechanics, 24:139-158, (1989).

8. P.F. Pai and A.H. Nayfeh, International Journal of NonLinear Mechanics, 25:455-474, (1990).

9. M.R.M. Crespo da Silva and C.L. Zaretzky, Nonlinear Dynamics, 5:3-23, (1994).

10. C.L. Zaretzky and M.R.M. Crespo da Silva, Nonlinear Dynamics, 5:161-180, (1994).

11. H.N. Arafat, A.H. Nayfeh, and C-M. Chin, Nonlinear Dynamics, 15:31-61(31), (1998).

12. N. Kacem, J. Arcamone, F. Perez-Murano, and S. Hentz, Journal of Micromechanics and Microengineering, 20(4):045023, (2010).

13. N. Kacem, S. Hentz, D. Pinto, B. Reig, and V. Nguyen, Nanotechnology, 20(27):275501, (2009).

14. N. Kacem and S. Hentz, Applied Physics Letters, 95(18):183104, (2009).

15. E. Mile, G. Jourdan, I. Bargatin, S. Labarthe, C. Marcoux, P. Andreucci, S. Hentz, C. Kharrat, E. Colinet, and L. Duraffourg, Nanotechnology, 21(16):165504, (2010)

16. K. Jensen, K. Kim and A. Zettl, Nature Nanotechnology, 3:533-537, (2008).

17. M.R.M. Crespo Da Silva, International Journal of Solids and Structures, 24:1225-1234, (1988).

18. M.R.M. Crespo Da Silva, International Journal of Solids and Structures, 24:1235-1242, (1988).

19. H. Nishiyama and M. Nakamura, IEEE Transactions on Components, Hybrids, and Manufacturing Technology, , 13(2):417-423, (1990).

20. C.Gui, R.Legtenberg, H.A.C. Tilmans, J.H.J. Fluitman, and M. Elwenspoek, Journal of Microelectromechanical Systems, 7(1):122-127, (1998).

21. L.C. Shao, M. Palaniapan, and W.W. Tan, Journal of Micromechanics and Microengineering, 18(6):065014, (2008).

22. V.A. Sazonova, A Tunable Carbon Nanotube Resonator. PhD thesis, Cornell University, (2006).

23. A.H. Nayfeh, M.I. Younis, and E.M. Abdel-Rahman, Nonlinear Dynamics, 48:153-163, (2007).

24. A. Boisen, Nature Nanotechnology, 4:404-405, (2009).

25. A.K. Naik, M.S. Hanay, W.K. Hiebert, X.L. Feng, and M.L. Roukes, Nature Nanotechnology, 4:445-449, (2009). 